Institute for Combustion Science and Environmental Technology 2413 Nashville Road, Bowling Green, KY 42101 Tel: (270) 745-2220 Fax: (270) 745-2221

\title{
Establishment of an Environmental Control Technology laboratory with a Circulating Fluidized-Bed Combustion System
}

Quarterly Technical Progress Report

October 1- December 31, 2006

Wei-Ping Pan, Zhongxian Cheng, Yan Cao and John Smith

January 2007

Cooperative Agreement No. DE-FC26-03NT41840

Institute for Combustion Science and Environmental Technology

Western Kentucky University

2413 Nashville Road

Bowling Green, KY 42101 


\section{DISCLAIMER}

This report was prepared as an account of work sponsored by an agency of the United States Government. Neither the United States Government nor any agency thereof, nor any of their employees, makes any warranty, express or implied, or assumes any legal liability or responsibility for the accuracy, completeness, or usefulness of any information, apparatus, product, or process disclosed, or represents that its use would not infringe privately owned rights. Reference herein to any specific commercial product, process, or service by trade name, trademark, manufacturer, or otherwise does not necessarily constitute or imply its endorsement, recommendation, or favoring by the United States Government or any agency thereof. The views and opinions of authors expressed herein do not necessarily state or reflect those of the United States Government or any agency thereof. 


\begin{abstract}
This report is to present the progress made on the project entitled "Establishment of an Environmental Control Technology Laboratory (ECTL) with a Circulating Fluidized-Bed Combustion (CFBC) System" during the period October 1, 2006 through December 31, 2006. The effort has been primarily concentrated on installation of CFBC Facility and preparation for cold fluidization operation. The assembly of the ash recirculation path from the cyclones back to the bed area of the Combustor has nearly been completed. Wind box assembly, including ash "drain"oulet, cast refractory "bowl" under the bubble nozzles and bed pre-heater has also been completed. Materials for flue gas duct from secondary cyclone to induced draft fan inlet have been specified and ordered. A laboratory-scale waste incineration test was conducted with two waste pellets. The purpose of this test is to characterize the by-products generated during the incineration.
\end{abstract}




\section{TABLE OF CONTENTS}

DISCLAIMER $\ldots \ldots \ldots \ldots \ldots \ldots \ldots \ldots \ldots$

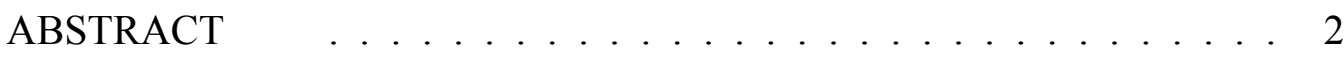

TABLE OF CONTENTS $\ldots \ldots \ldots \ldots \ldots \ldots$

LIST OF FIGURES . . . . . . . . . . . . . . . . . . . . . . . 4

LIST OF TABLES $\ldots \ldots \ldots \ldots \ldots \ldots \ldots$

1. EXECUTIVE SUMMARY $\ldots \ldots \ldots \ldots \ldots \ldots$

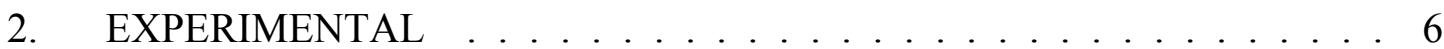

2.1 Installation of the CFBC Facility. . . . . . . . . . . . . . . 6

2.2 Experimental Study of Air Pollutant Emission by Optimized

Combustion in a Laboratory-Scale Incinerator. . . . . . . . . . . . 9

3. RESULTS AND DISCUSSION . . . . . . . . . . . . . . 14

3.1 Gaseous Air Pollutants Generated during Tests in the Laboratory-Scale Incinerator. . . . . . . . . . . . . . . . . . . . . . . . . 14

3.2 TCLP Pollutants in Fly Ash and Bottom Ash Generated during Tests in the Laboratory-Scale Incinerator. . . . . . . . . . . . . . . . . . . . . . 18

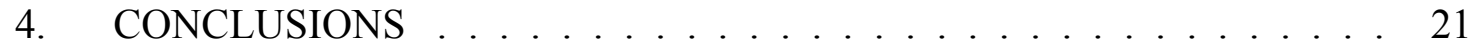

5. FUTURE WORK AND UPATED SCHEDULE . . . . . . . . . . . . 21

5.1 Future Work. . . . . . . . . . . . . . . . . . . . . . 21

5.2 Project Schedule. . . . . . . . . . . . . . . . . . . . . . 22

ACRONYMS AND ABBREVIATIONS . . . . . . . . . 23 


\section{LIST OF FIGURES}

Figure 1. Photograph of the Laboratory-Scale Waste Incinerator . . . . . . 10

Figure 2. IMR5000 Flue Gas Monitor. . . . . . . . . . . . . . . . . 11

Figure 3. EPA Method 29 for Trace Metals Sampling. . . . . . . . . . . 12

Figure 4. EPA Method 0033 for VOC Sampling. . . . . . . . . . . . . 13

Figure 5. EPA Method 0011 for Semi-VOC Sampling. . . . . . . . . . . 13 


\section{LIST OF TABLES}

Table 1. CFBC Construction Progress Information . . . . . . . . . . 7

Table 2. Lists of EPA Methods Used. . . . . . . . . . . . . . . . . . 14

Table 3. Emissions of Gaseous Air Pollutants. . . . . . . . . . . . . 15

Table 4. Emissions of Gaseous VOC . . . . . . . . . . . . . . 16

Table 5. Trace Metals in Fly Ash from Waste Pellets after Incineration by TCLP .................................. 19

Table 6. Trace Metals in Bottom Ash from Waste Pellets after Incineration

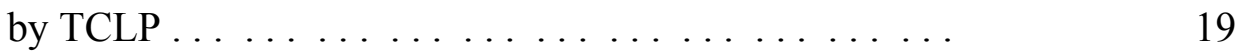

Table 7. VOC in Fly Ash from Waste Pellets after Incineration by TCLP . . 20

Table 8. Semi-VOC in Fly Ash from Waste Pellets after Incineration by TCLP ............................. 20

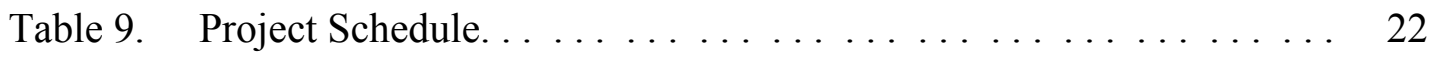




\section{EXECUTIVE SUMMARY}

The effort has been primarily concentrated on preparation for cold fluidization operation, although progress has been made on other tasks. The assembly of the ash recirculation path from the cyclones back to the bed area of the Combustor has nearly been completed. Wind box assembly, including ash "drain"oulet, cast refractory "bowl" under the bubble nozzles and bed pre-heater has also been completed. The secondary combustion air blower and variable speed drive have been received and are ready for installation and connection to the four levels of secondary air injection. Further, the induced draft fan has been ordered and is due to arrive on January $31^{\text {st }}, 2007$. Finally, materials for the flue gas duct from secondary cyclone to induced draft fan inlet have been specified and ordered.

A laboratory-scale waste incineration test was conducted with two waste pellets. The purpose of this test was to characterize the by-products generated during the incineration.

\section{EXPERIMENTAL}

\subsection{Installation of the CFBC Facility}

Installation of the circulating fluidized-bed combustor (CFBC) continues during this period. The specific tasks completed during this period are:

a) All ash recirculation ducts have been aligned and installed, including four thermal expansion joints and duct support hardware.

b) Both loop seals have been installed in ash recirculation path.

c) Cold ash supply duct has been installed, including one thermal expansion joint.

d) Induced draft fan has been specified, ordered and is now forecast to arrive on site January 31,2007 . The shaft seal manufacturer had problems producing the required shaft seal and delayed the fan manufacturer's delivery for more than a month.

e) Installation of the induced draft fan power supply and variable speed drive has been completed.

f) Secondary combustion air blower has been received and it's power supply and variable speed drive installation is complete. 
g) Secondary combustion air distribution control valves have been received.

h) Flue duct from secondary cyclone to induced draft fan inlet and the induced draft fan discharge duct have been designed, components specified and ordered.

i) Wind box installation, including cast refractory "bowl" that helps shape fluidization pattern at bubble plate nozzles has been completed.

j) Lower level bed ash drain outlet has been completed. Rapid bed ash drain flange has been capped with a blind flange.

k) Assembly of primary combustion/fluidizing air inlet duct from supply blowers to wind box has been completed, including a bed pre-heater diverter valve.

1) Assembly of bed pre-heater that heats the fluidizing air just prior to entering the windbox is nearly complete.

m) Sensor and control signal cable raceway enclosure at control room has been completed that allows these cables to connect to the process control and data logging computer in control room.

n) Many coolant system components have been specified and ordered, including heat exchanger flow control valves, pumps, expansion tank, gas/liquid separator and major water supply piping.

Table 1 lists all construction tasks and the estimated percent complete for each.

Table 1. CFBC Construction Progress Information

\begin{tabular}{|l|l|c|}
\hline \multicolumn{1}{|c|}{ Task Area } & \multicolumn{1}{c|}{ Specific Activities } & $\begin{array}{c}\text { Estimated } \\
\text { Percent } \\
\text { Completed }\end{array}$ \\
\hline \hline Ash (Hot \& Cold) Supply Ducts & \multicolumn{1}{c|}{} & \multicolumn{1}{c|}{} \\
\hline Primary Cyclone Support & Assemble Ducts, Solids Bend, Thermal Exp. Joints, Loop Seals & $\mathbf{1 0 0}$ \\
\hline Secondary Cyclone Support & Assemble support, align to ash ducts and secondary cyclone & $\mathbf{1 0 0}$ \\
\hline Fuel/Bed Material Supply Ducts & Assemble support, align to ash ducts & $\mathbf{1 0}$ \\
\hline ID Fan & Assemble Ducts, Rotary Valves, Windows, Thermal Exp. Joints & $\mathbf{9 0}$ \\
\hline ID Fan Variable Speed Drive (VSD) & Parameters established, Initiate RFQs, Purchase, Install & $\mathbf{1 0 0}$ \\
\hline Lower Loop Seal Flow Control & Parameters established, Initiate RFQs, Purchase, Install & $\mathbf{5}$ \\
\hline Upper Loop Seal Flow Control & Determine required control properties & $\mathbf{5}$ \\
\hline Secondary Combustion Air Blowers & Determine required control properties & $\mathbf{1 0 0}$ \\
\hline Secondary Air Variable Speed Drives (VSD) & Confirm Parameters, Initiate RFQs, Purchase & $\mathbf{1 0 0}$ \\
\hline Secondary Air Control Valves & Establish Parameters, Initiate RFQs, Purchase, Install & $\mathbf{7 0}$ \\
\hline Ash Bunker Auger & Establish Parameters, Initiate RFQs, Purchase, Install & $\mathbf{4 0}$ \\
\hline
\end{tabular}




\begin{tabular}{|c|c|c|}
\hline Ash Bunker Auger Drive & Confirm Parameters, Initiate RFQs, Purchase, Install & 40 \\
\hline Ash Bunker Auger Power Supply & Establish Parameters, Initiate RFQs, Purchase, Install & 20 \\
\hline Ash Supply Auger Tube & Determine if on site, proceed from this finding & 5 \\
\hline Ash Supply Bunker Auger & Mass Flow establised, Establish other Parameters, Intiate RFQs & 10 \\
\hline Flue Duct: Secondary Cyclone to ID Fan & Duct: Design, Specify, Purchase, Install & 35 \\
\hline ID Fan Discharge Duct & Duct: Design, Specify, Purchase, Install & 70 \\
\hline Lower Loop Seal Flow Control & Provide controlled compressed air, piping \& computer control & 5 \\
\hline Upper Loop Seal Flow Control & Provide controlled compressed air, piping \& computer control & 5 \\
\hline Pressure \& Temperature Sensors & Specify Components, Purchase & 70 \\
\hline Rotary Air Lock Valve Drives & Determine Locations, Specify, Initiate RFQs, Purchase & 10 \\
\hline Rotary Air Lock Valve Drive Power Supplies & Parameters established, Initiate RFQs, Purchase, Install & 80 \\
\hline Fuel Duct Window for Flow Proving Sensors & Complete Design, Acquire Materials, Construct & 10 \\
\hline Secondary Combustion Air Blowers & Establish locations for each, Install & 70 \\
\hline Secondary Combustion Air VSD & Parameters established, Initiate RFQs, Purchase, Install & 100 \\
\hline Secondary Air Blower Power Supplies & Parameters established, Initiate RFQs, Purchase, Install & 100 \\
\hline Supply Bunker Augers (3 ea.) & Mass Flow establised, Establish other Parameters, Intiate RFQs & 10 \\
\hline Supply Transverse Auger & Mass Flow establised, Establish other Parameters, Intiate RFQs & 10 \\
\hline Supply Transverse Auger Power Supply & Establish parameters, initiate RFQs & 50 \\
\hline Supply Bunker Auger Drive Power Supplies & Parameters established, Initiate RFQs, Purchase, Install & 10 \\
\hline Power Supplies for all Computer Interfaces & Determine Location, Specify, Initiate RFQs, Purchase & 5 \\
\hline Un-used Ports & Identify \& Specify Closures for all & 5 \\
\hline Ash Bunker Auger Drive Control & Signal cable, conduit: Specify, Purchase, Install & 10 \\
\hline Ash Bunker Auger Drive Control Interface & Install in Enclosure, connect control \& data cable & 5 \\
\hline Ash Bunker Auger Drive Interface Enclosure & Determine Location, Specify, Purchase, Install & 5 \\
\hline Ash Supply Bunker Support & Develop Support Design, Acquire Materials,Construct & 20 \\
\hline Bubble Plate Refractory Molding & Research geometry \& materials; Apply to Plate; Assemble Plate & 40 \\
\hline Cargo Lift System & Complete Design, Purchase Supplies, Construct & 30 \\
\hline FD Fan VSD Control & Signal cable, conduit: Specify, Purchase, Install & 5 \\
\hline FD Fan VSD Control Interface & Install in Enclosure, connect control \& data cable & 5 \\
\hline Fluidizing Air/Bed Preheater & Parameters established, Initiate RFQs, Purchase, Install & 70 \\
\hline ID Fan Control & Computer Control \& Display Program & 85 \\
\hline ID Fan VSD Control & Signal cable, conduit: Specify, Purchase, Install & 5 \\
\hline ID Fan VSD Control Interface & Install in Enclosure, connect control \& data cable & 5 \\
\hline ID Fan VSD Control Interface Enclosure & Determine Location, Specify, Purchase, Install & 5 \\
\hline Pressure \& Temperature Sensors & Modify Riser Ports/ Surrounding Floor Frame, Install & 15 \\
\hline Rotary Air Lock Valve Drive Controls & Signal cable, conduit: Specify, Purchase, Install & 5 \\
\hline Rotary Air Lock Valve Drive Interfaces & Install in Enclosure, connect control \& data cable & 5 \\
\hline Rotary Valve Drive Interface Enclosures & Determine Location, Specify, Purchase, Install & 5 \\
\hline Secondary Air Blower Controls & Computer Control \& Display Program & 85 \\
\hline Secondary Air Blower VSD Controls & Signal cable, conduit: Specify, Purchase, Install & 5 \\
\hline Secondary Air Blower VSD Control Interfaces & Install in Enclosure, connect control \& data cable & 5 \\
\hline Sec. Air Blower Control Interface Enclosures & Determine Location, Specify, Purchase, Install & 5 \\
\hline Secondary Air Blower Discharge Ducts & Specify material(s), size, locations & 50 \\
\hline Sensor Signal & Signal cable, conduit: Specify, Purchase, Install & 5 \\
\hline Sensor Signal Interface & Install in Enclosure, connect control \& data cable & 5 \\
\hline Sensor Signal Interface Enclosure & Determine Locations, Specify, Purchase, Install & 5 \\
\hline Windbox \& Ash Drain & Determine Ash Drain Sliding Seal Design, Assemble Windbox & 70 \\
\hline Fluidizing Air Supply & Assemble flexible duct from Blower Duct to Windbox inlet & 80 \\
\hline
\end{tabular}




\begin{tabular}{|c|c|c|}
\hline Supply Bunker Supports & Complete Design, Purchase Supplies, Construct & 20 \\
\hline Supply Bunker Augers (3 ea.) & Install Augers, Seals \& Support Bearings & 10 \\
\hline Supply Transverse Auger Drive & Parameters established, Initiate RFQs, Purchase, Install & 10 \\
\hline Transverse Auger Tube to Rotary Valve Adpt. & Design and Construct corrected adapter allowing connection & 10 \\
\hline All Other Sensors & Parameters established, Initiate RFQs & 40 \\
\hline Supply Bunker Auger Tubes Assembly & Determine if on site, proceed from this finding & 10 \\
\hline Supply Bunker Auger Drive Interfaces & Install in Enclosure, connect control \& data cable & 10 \\
\hline Supply Bunker Auger Drive Interface Encl. & Determine Location, Specify, Purchase, Install & 10 \\
\hline Supply Flow Proving Sensors & Design Complete, Specify Components... & 20 \\
\hline Supply Transverse Auger & Install Auger, Seal \& Support Bearings & 10 \\
\hline Supply Transverse Auger Tube & Determine if on site, proceed from this finding & 0 \\
\hline Supply Transverse Auger Drive Controls & Signal cable, conduit: Specify, Purchase, Install & 10 \\
\hline Supply Transverse Auger Drive Interfaces & Install in Enclosure, connect control \& data cable & 10 \\
\hline $\begin{array}{l}\text { Supply Transverse Auger Drive Interface } \\
\text { Encl. }\end{array}$ & Determine Location, Specify, Purchase, Install & 10 \\
\hline Visual Display of all other Process Variables & Design Complete, Specify Components... & 75 \\
\hline Process Meas. \& Control System & Correct/Modify as Required; Otherwise nearly Complete & 95 \\
\hline Ash (Hot \& Cold) Supply Ducts & All Ash Ducts on site & 100 \\
\hline Ash Supply Bunker & Ash Supply Bunker on site & 100 \\
\hline Fuel/Bed Material Supply Ducts & All Supply Ducts on site & 100 \\
\hline Rotary Air Lock Valves, Motors (2 ea.) & All Rotary Valves, Motors on site & 100 \\
\hline Supply Bunker Auger Drive Controls & All Supply Drive Controls on site & 100 \\
\hline Supply Bunker Auger Drives (3 ea.) & All Supply Drives on site & 100 \\
\hline Supply Bunker Load Cells & All Load Cells on site & 100 \\
\hline Supply Bunkers & All Supply Bunkers on site & 100 \\
\hline Heat Exchangers & Coolant control Valves: Specify, Purchase, Install & 70 \\
\hline
\end{tabular}

\subsection{Experimental Study of Air Pollutant Emissions by Optimized Combustion in a}

\section{Laboratory-Scale Incinerator}

\subsubsection{Incinerator}

A laboratory-scale tube flow incinerator was used to evaluate the combustion performance of waste pellets and the resulting emissions. A photograph of the tube flow incinerator is shown in Figure 1. The incinerator is made of a 2" I.D. stainless steel pipe and is a 4-foot long. A thermocouple is inserted into the reactor to monitor the internal temperature. Considering that high temperature is desirable for the decomposition of long carbon chain organics, a two-stage furnace with a two-channel temperature controller is used to heat the incinerator. Thus, the desired temperature can be achieved at different locations inside the incinerator. During incineration with waste pellets, temperature in the upper part of the incinerator is maintained higher than that at the bottom part in order to decompose Volatile Organic Compounds (VOC) and Semi-volatile Organic Compounds 
(Semi-VOC) with the high carbon dioxide $\left(\mathrm{CO}_{2}\right)$ concentration off gas generated by high heating rates. The testing samples were waste material which had been used for gas generator of vehicle air bag. They look like small balls and the diameter falls in the range of $2 \mathrm{~mm}$. During incineration, two waste pellet samples (called NQ-AD and QB-B) were fed into the incinerator in individual tests by a mini screw feeder at a rate of $3.9 \mathrm{~g} / \mathrm{min}$. Air is blown into the incinerator with an excess air ratio of 1.8 to ensure better incineration. The incineration conditions are shown as follows:

Feeding rate: $3.9 \mathrm{~g} / \mathrm{min}$;

Air: $10 \mathrm{l} / \mathrm{min}$ (minimum air flow rate for complete incineration is $5.5 \mathrm{l} / \mathrm{min}$ );

Temperature: $800{ }^{\circ} \mathrm{C}$ (top); $600{ }^{\circ} \mathrm{C}$ (bottom);

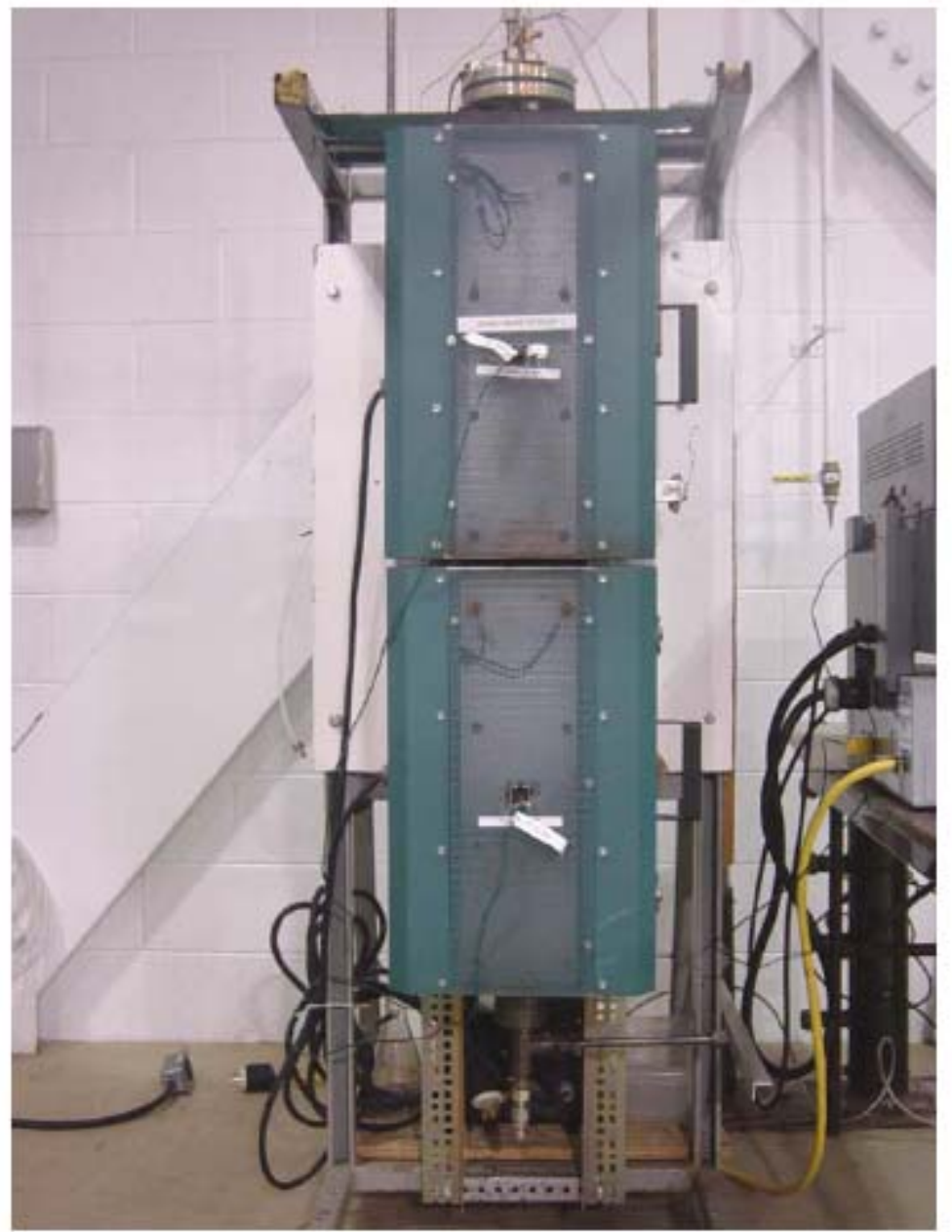

Figure 1. Photograph of the laboratory-scale waste incinerator 


\subsubsection{Instrumentation and Flue Gas Monitor}

The gaseous products exit the outlet at the top end of the incinerator to instrument or sampling trains. All sampling lines are made of Teflon material to prevent contamination and ensure measurement accuracy. An IMR5000 gas analyzer, as shown in Figure 2, is used to monitor the flue gas composition including oxygen $\left(\mathrm{O}_{2}\right)$, methane $\left(\mathrm{CH}_{4}\right)$, ammonia $\left(\mathrm{NH}_{3}\right)$, hydrogen sulfide $\left(\mathrm{H}_{2} \mathrm{~S}\right)$, hydrogen chloride $(\mathrm{HCl})$, carbon dioxide $\left(\mathrm{CO}_{2}\right)$, carbon monoxide $(\mathrm{CO})$, sulfur dioxide $\left(\mathrm{SO}_{2}\right)$, chlorine $\left(\mathrm{Cl}_{2}\right)$, nitrogen monoxide (NO) and nitrogen dioxide $\left(\mathrm{NO}_{2}\right) . \mathrm{NO}_{\mathrm{x}}\left(\mathrm{NO}, \mathrm{NO}_{2}\right)$ species was also sampled by an impinger train, which is analyzed by DX-120 Ion Chromatograph Analyzer based on EPA Method 7d.

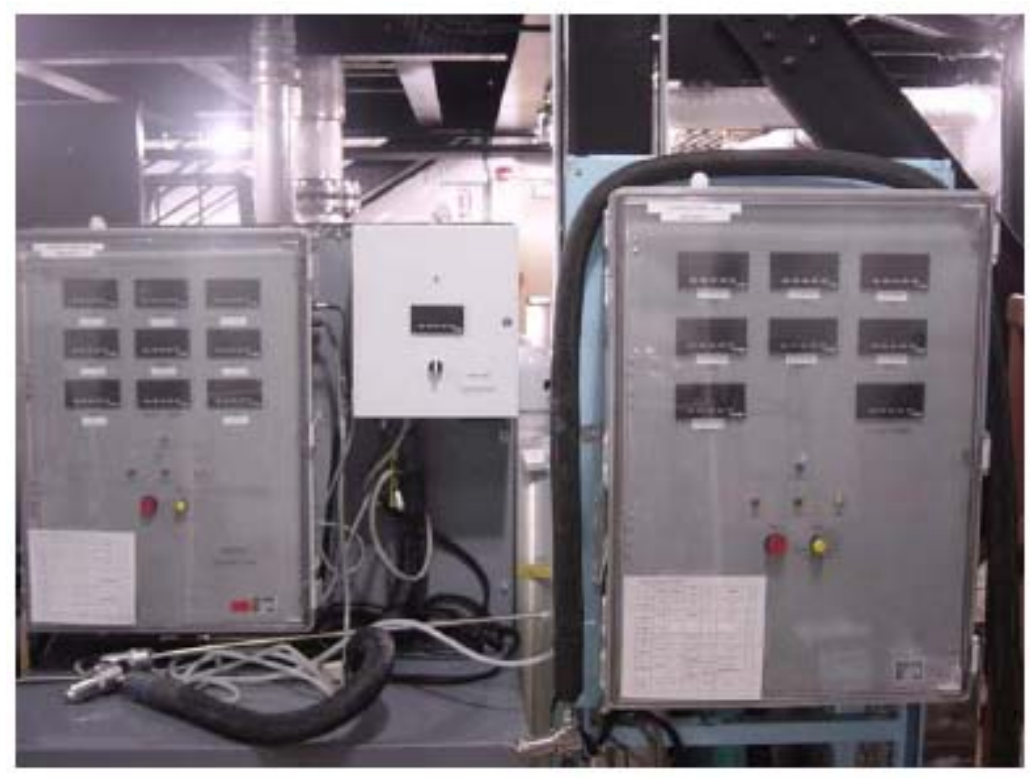

Figure 2. IMR5000 flue gas monitor

\subsubsection{EPA Methods Sampling Train}

The determination of Particulate Matter (PM) emission rates is based on EPA Method 17. EPA Method 29, as shown in Figure 3, is used to characterize gaseous trace metals (arsenic, chromium, cadmium, lead, iron, mercury, nickel, silver, copper and strontium). EPA method 1311 (Toxicity Characteristic Leaching Procedure, TCLP) is 
used to characterize trace metals by Inductively Coupled Plasma - Atomic Emission Spectrometer (ICP-AES) for all metals except mercury. Leeman cold-vapor atomic absorption (CVAA) is used for mercury determination. EPA Method 0030 and Method 0010, as shown in Figures 4 and 5, are used to characterize VOCs and Semi-VOCs, respectively. VOC analysis includes benzene, carbon tetrachloride, chlorobenzene, chloroform, 1,2-dichloroethane, 1,1-dichloroethene, methyl ethyl ketone, tetrachloroethene, trichloroethene, and vinyl chloride. Semi-volatile organic compounds include cresols, 1,4-dichlorobenzene, 2,4-dinitrotoluene, hexachlorobenzene, hexachlorobutadiene, hexachloroethane, nitrobenzene, pentachlorophenol, pyridine, 2,4,5-trichlorophenol, and 2,4,6-trichlorophenol. Volatile and semi-volatile organic compounds in particulate matter and burn residue are analyzed according to EPA Method 1311. The detailed EPA methods used for these characterizations are listed in Table 2.

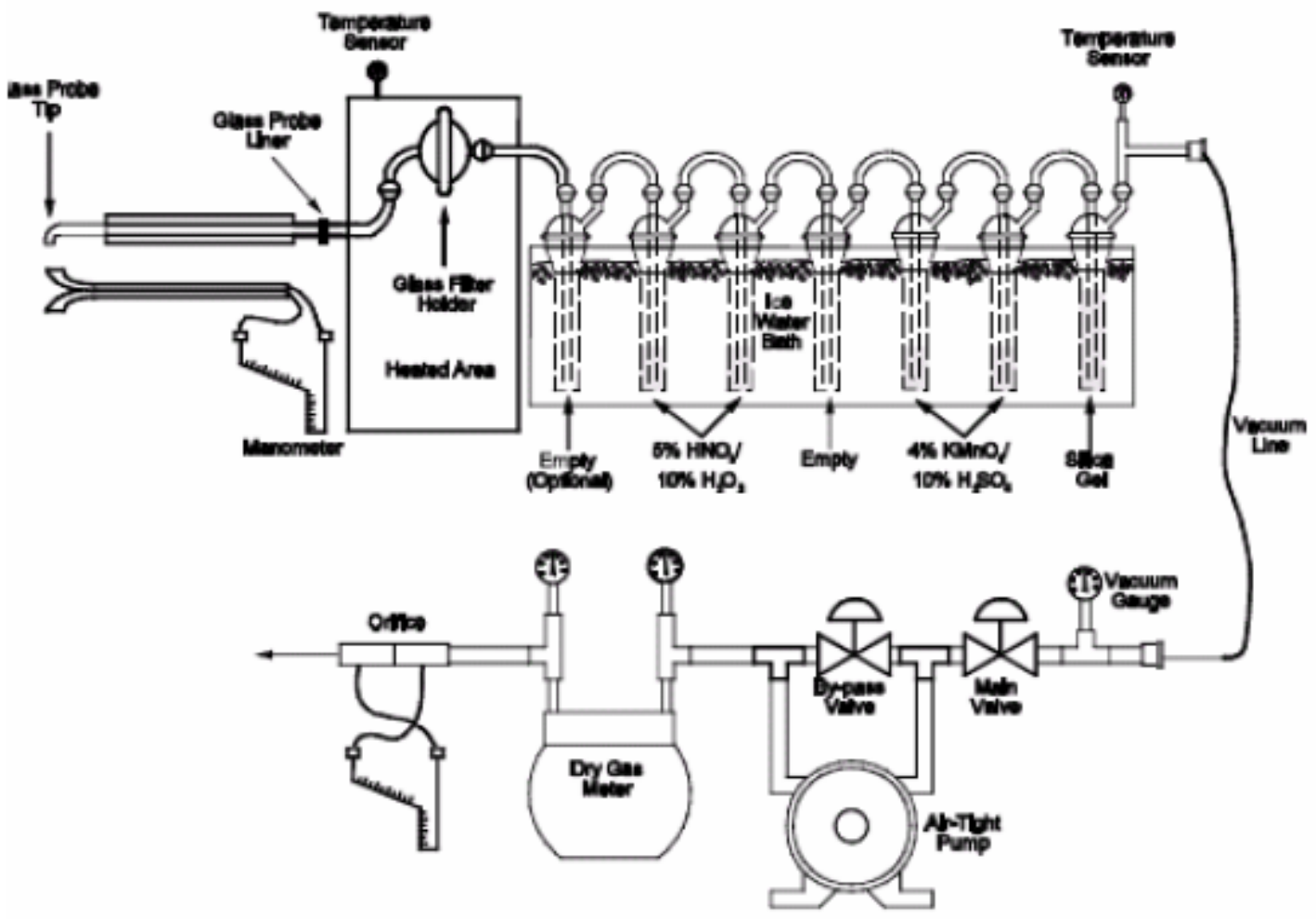

Figure 3. EPA Method 29 for trace metals sampling 


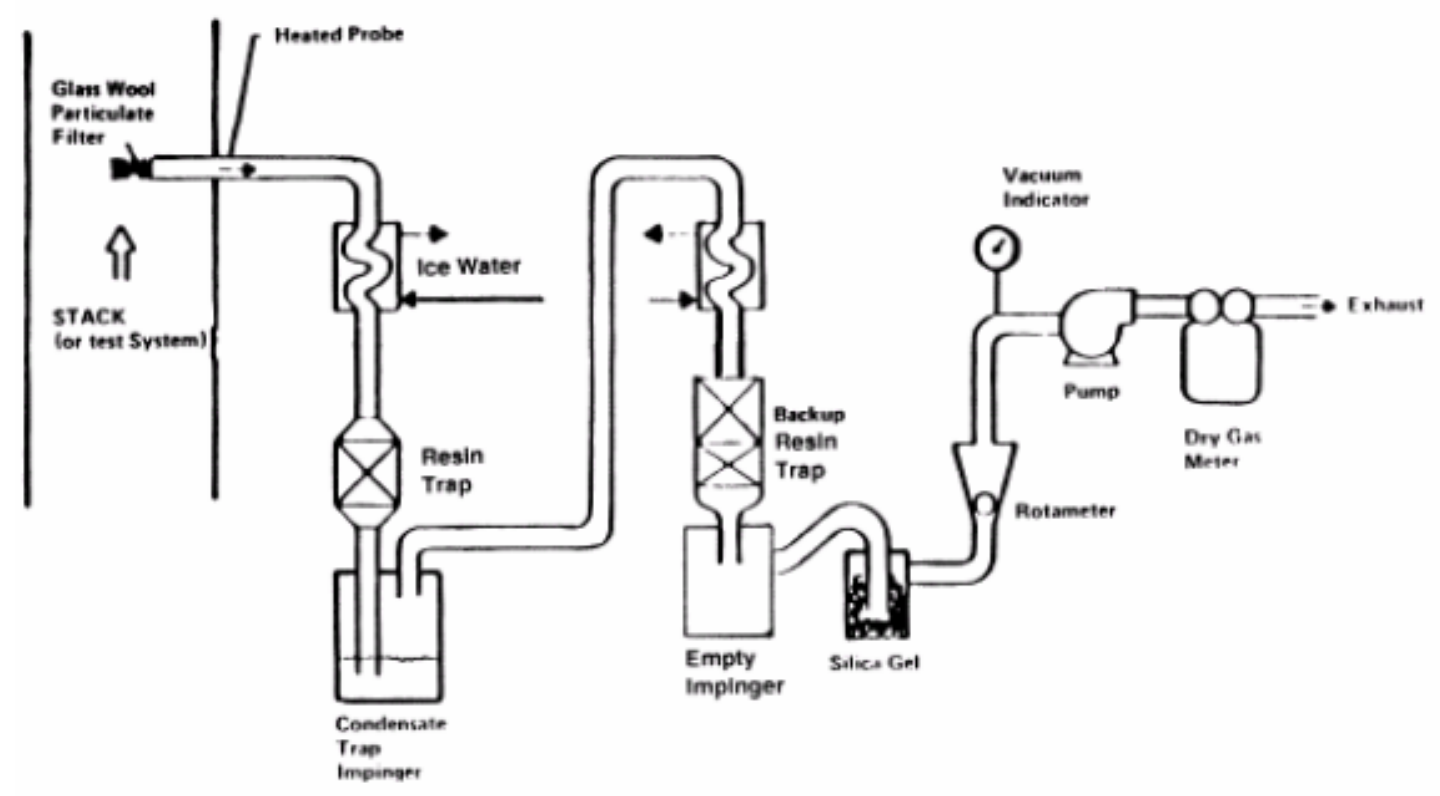

Figure 4. EPA Method 0033 for VOC sampling

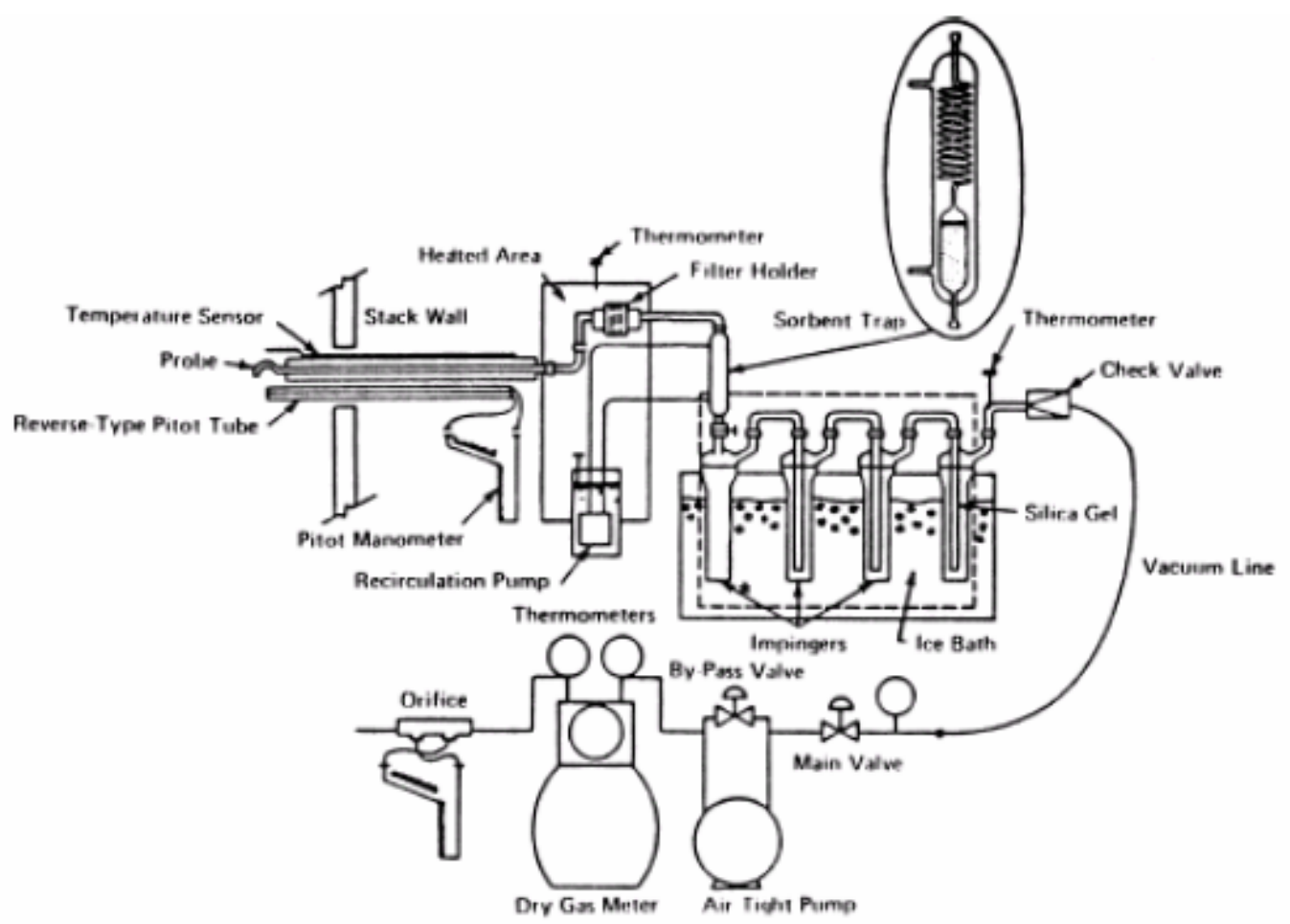

Figure 5. EPA Method 0011 for semi-VOC sampling 
Table 2. Lists of EPA Methods used

\begin{tabular}{|c|c|c|c|}
\hline \multicolumn{4}{|l|}{ EPA Methods } \\
\hline $\begin{array}{c}\text { Trace metals } \\
\text { Sampling } \\
\text { Sample extraction } \\
\text { Analysis } \\
\end{array}$ & $\begin{array}{c}\text { Off gas } \\
\text { EPA M-29 } \\
- \\
\text { EPA } 200.7 \\
\end{array}$ & $\begin{array}{c}\text { Fly ash } \\
\text { EPA M-29 } \\
\text { EPA TCLP } 1311 \\
\text { EPA } 200.7 \\
\end{array}$ & $\begin{array}{l}\text { Bottom ash } \\
\text { Grab sampling method } \\
\text { EPA TCLP } 1311 \\
\text { EPA 200.7 } \\
\end{array}$ \\
\hline $\begin{array}{c}\text { VOC } \\
\text { Sampling } \\
\text { Sample extraction } \\
\text { Analysis } \\
\end{array}$ & $\begin{array}{c}\text { Off gas } \\
\text { EPA 0030 } \\
\text { EPA Thermal desorbtion Method } 5041 \\
8260 \mathrm{~B} \\
\end{array}$ & $\begin{array}{c}\text { Fly ash } \\
\text { Grab Sampling Method } \\
\text { EPA TCLP } 1311 \\
8260 \mathrm{~B} \\
\end{array}$ & $\begin{array}{c}\text { Bottom ash } \\
\text { Grab Sampling Method } \\
\text { EPA TCLP } 1311 \\
8260 \mathrm{~B} \\
\end{array}$ \\
\hline $\begin{array}{c}\text { Semi-VOC } \\
\text { Sampling } \\
\text { Sample extraction } \\
\text { Analysis } \\
\end{array}$ & $\begin{array}{l}\text { Off gas } \\
\text { EPA } 0010 \\
\text { EPA } 3541 \\
\text { EPA } 8270 \text { D }\end{array}$ & $\begin{array}{c}\text { Fly ash } \\
\text { Grab Sampling Method } \\
\text { EPA TCLP } 1311 \\
\text { EPA } 8270 \text { D } \\
\end{array}$ & $\begin{array}{l}\text { Bottom ash } \\
\text { Grab Sampling Method } \\
\text { EPA TCLP } 1311 \\
\text { EPA 8270 D } \\
\end{array}$ \\
\hline
\end{tabular}

\section{RESULTS AND DISCUSSION}

\subsection{Gaseous Air Pollutants Generated during Tests in the Laboratory-Scale Incinerator}

Results from the laboratory-scale waste incinerator indicate that nitrogen species are the main air pollutants generated during incineration of waste pellets. Table 3 presents the emission results for nitrogen species. Results indicated that sample QB-B emits a higher concentration of nitrogen compounds than sample NQ-AD. For example, NO and $\mathrm{NO}_{2}$ emissions from sample QB-B varied between 2000-2200 ppm and 530-570 ppm, respectively, versus 520-550 ppm of $\mathrm{NO}$ and 530-570 ppm of $\mathrm{NO}_{2}$ for sample NQ-AD. However, $\mathrm{N}_{2} \mathrm{O}$ emissions from sample QB-B is lower between 245-267 ppm, than that from sample NQ-AD, which ranged between 880-1013 ppm. $\mathrm{NH}_{3}, \mathrm{CO}$, and $\mathrm{CH}_{4}$ were not detected because excess air is provided to maintain complete incineration during test. These compounds typically exist in an oxygen limited environment, which was not an issue in this work.

The emission rates of gaseous VOCs is largely controlled, as shown in Table 4. The majority of the VOC species are less than the detection limit, except for benzene. The benzene emission rate varied from $0.47 \mu \mathrm{g}(0.27+0.209$ for trap 1 and trap 2 in series $)$ to $1.031 \mu \mathrm{g}(0.78+0.251$ for trap 1 and trap 2 in series $)$ for the sample NQ-AD, and from 
$1.041 \mu \mathrm{g}$ to $1.41 \mu \mathrm{g}$ for the sample QB-B. Methane ethyl ketone present at a level of 0.5 $\mu \mathrm{g}$ is found during incineration of the sample QB-B, but not for parallel testing with sample NQ-AD. Benzene levels can be easily controlled by increasing reactor temperature (high combustion temperature with sufficient oxygen will reduce the formation of benzene). An open combustion configuration could produce less $(<1 \mu \mathrm{g})$ benzene than was found in this work. Test results for all gaseous semi-VOCs are found to be less than the detection limit $(0.01 \mu \mathrm{g})$ for all semi-VOC compounds specified in EPA Method 0011. The specific semi-VOCs should be completely decomposed during the incineration process.

Table 3. Emissions of Gaseous Air Pollutants

\begin{tabular}{lcccc}
\hline \multicolumn{5}{c}{ Nitrogen species } \\
\hline & $\mathrm{NO}$ & $\mathrm{NO}_{2}$ & $\mathrm{~N}_{2} \mathrm{O}$ & $\mathrm{NH}_{3}$ \\
\hline \multicolumn{5}{c}{ In ppm } \\
\hline $\mathrm{NQ}-\mathrm{AD}$ & $518-546$ & $229-236$ & $879-1013$ & $\mathrm{ND}$ \\
\hline $\mathrm{QB}-\mathrm{B}$ & $2020-2211$ & $529-567$ & $245-267$ & $\mathrm{ND}$ \\
\hline \multicolumn{5}{c}{ Other combustion source species } \\
& $\mathrm{CO}$ & $\mathrm{CH}_{4}$ & $\mathrm{SO}_{2}$ & $\mathrm{HCl}$ \\
\hline $\mathrm{NQ}-\mathrm{AD}$ & $\mathrm{ND}$ & $\mathrm{ND}$ & $\mathrm{ND}$ & $\mathrm{ND}$ \\
\hline QB-B & $\mathrm{ND}$ & $\mathrm{ND}$ & $\mathrm{ND}$ & $\mathrm{ND}$ \\
\hline
\end{tabular}

Notes: Estimated product gas generation $=3 \mathrm{~L} / \mathrm{g}$ raw waste pellet, thus, $1 \mathrm{NM} 3 / 0.33 \mathrm{Kg}$ raw waste pellets 
Table 4. Emissions of Gaseous VOC

\begin{tabular}{|c|c|c|c|c|c|c|c|}
\hline & Units & VOST-BLANK & RDL & QC Batch & \begin{tabular}{|c|} 
VOC-1 NQ \\
AD-19/17/06 \\
\end{tabular} & RDL & QC Batch \\
\hline Vinyl Chloride & ug & ND & 0.01 & 1056763 & ND & 0.1 & 1057747 \\
\hline 1.1-Dichloroethane & ug & ND & 0.01 & 1056763 & ND & 0.1 & 1057747 \\
\hline Chloroform & ug & ND & 0.01 & 1056763 & ND & 0.1 & 1057747 \\
\hline 1,2-Dichloroethane & ug & ND & 0.007 & 1056763 & ND & 0.07 & 1057747 \\
\hline Methyl Ethyl Ketone (2-Butanone) & ug & ND & 0.04 & 1056763 & ND & 0.4 & 1057747 \\
\hline Carbon Tetrachloride & ug & ND & 0.02 & 1056763 & ND & 0.2 & 1057747 \\
\hline Benzene & ug & 0.015 & 0.009 & 1056763 & 0.78 & 0.09 & 1057747 \\
\hline Trichloroethylene & ug & ND & 0.01 & 1056763 & ND & 0.1 & 1057747 \\
\hline Tetrachloroethylene & ug & ND & 0.02 & 1056763 & ND & 0.2 & 1057747 \\
\hline Chlorobenzene & ug & ND & 0.01 & 1056763 & ND & 0.1 & 1057747 \\
\hline \multicolumn{8}{|l|}{ Surrogate Recovery $(\%)$} \\
\hline Gromofluorobenzene & $\%$ & 96 & $N / A$ & 1056763 & 71 & $\mathrm{~N} / \mathrm{A}$ & 1057747 \\
\hline D4-1,2-Dichloroethane & $\%$ & 103 & N'A & 1056763 & 74 & $\mathrm{~N} / \mathrm{A}$ & 1057747 \\
\hline D8-Toluene & $\%$ & 96 & $N^{\prime} / A$ & 1056763 & 78 & $\mathrm{~N} / \mathrm{A}$ & 1057747 \\
\hline $\begin{array}{l}\text { ND = Not detected } \\
\text { N/A = Not Applicable } \\
\text { RDL = Reportable Detection Limit } \\
\text { QC Batch = Quality Control Batch }\end{array}$ & & & & & & & \\
\hline
\end{tabular}

\begin{tabular}{|c|c|c|c|c|c|c|c|}
\hline & Jnits & \begin{tabular}{|c|} 
VOC-1 NQ \\
AD-2 $9 / 17 / 06$ \\
\end{tabular} & RDL & QC Batch & \begin{tabular}{|c|c|} 
VOC-2 QA \\
ND-1 $9 / 17 / 06$ \\
\end{tabular} & RDL & QC Batch \\
\hline Vinyl Chloride & ug & ND & 0.01 & 1057747 & ND & 0.1 & 1058299 \\
\hline 1,1-Dichloroethane & ug & ND & 0.01 & 1057747 & ND & 0.1 & 1058299 \\
\hline Chloroform & ug & ND & 0.01 & 1057747 & ND & 0.1 & 1058299 \\
\hline 1,2-Dichloroethane & ug & ND & 0.007 & 1057747 & ND & 0.07 & 1058299 \\
\hline Methyl Ethyl Ketone (2-Butanone) & ug & ND & 0.04 & 1057747 & ND & 0.4 & 1058299 \\
\hline Carbon Tetrachloride & ug & $\mathrm{ND}$ & 0.02 & 1057747 & ND & 0.2 & 1058299 \\
\hline Benzene & ug & 0.251 & 0.009 & 1057747 & 0.27 & 0.09 & 1058299 \\
\hline Trichloroethylene & ug & ND & 0.01 & 1057747 & ND & 0.1 & 1058299 \\
\hline Tetrachloroethylene & ug & ND & 0.02 & 1057747 & ND & 0.2 & 1058299 \\
\hline Chlorobenzene & ug & ND & 0.01 & 1057747 & ND & 0.1 & 1058299 \\
\hline \multicolumn{8}{|l|}{ Surrogate Recovery (\%) } \\
\hline Bromofluorobenzene & $\%$ & 94 & N/A & 1057747 & 84 & N/A & 1058299 \\
\hline D4-1,2-Dichloroethane & $\%$ & 100 & NIA & 1057747 & 108 & N/A & 1058299 \\
\hline D8-Toluene & $\%$ & 94 & N/A & 1057747 & 99 & N/A & 1058299 \\
\hline \multicolumn{8}{|l|}{$\begin{array}{l}\text { ND = Not detected } \\
\text { N/A = Not Applicable } \\
\text { RDL = Reportable Detection Limit } \\
\text { QC Batch = Quality Control Batch }\end{array}$} \\
\hline
\end{tabular}




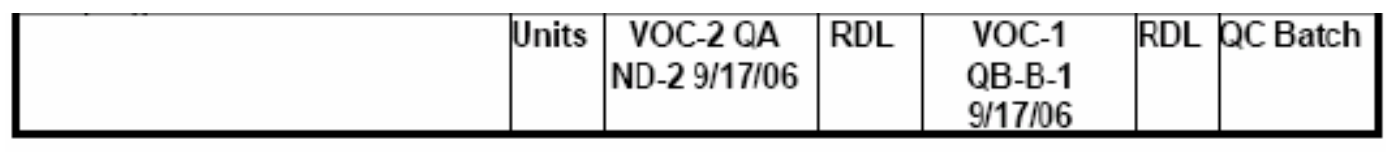

\begin{tabular}{|l|l|l|l|l|l|l|}
\hline Virnl Chloride & ug & ND & 0.01 & ND & 0.1 & 1057747 \\
\hline 1,1-Dichloroethane & ug & ND & 0.01 & ND & 0.1 & 1057747 \\
\hline Chloroform & ug & ND & 0.01 & ND & 0.1 & 1057747 \\
\hline 1,2-Dichloroethane & ug & ND & 0.007 & ND & 0.07 & 1057747 \\
\hline Methyl Ethyl Ketone (2-Butanone) & ug & ND & 0.04 & ND & 0.4 & 1057747 \\
\hline Carbon Tetrachloride & ug & ND & 0.02 & ND & 0.2 & 1057747 \\
\hline Benzene & ug & 0.209 & 0.009 & 0.67 & 0.09 & 1057747 \\
\hline Trichloroethylene & ug & ND & 0.01 & ND & 0.1 & 1057747 \\
\hline Tetrachloroethylene & ug & ND & 0.02 & ND & 0.2 & 1057747 \\
\hline Chlorobenzene & ug & ND & 0.01 & ND & 0.1 & 1057747 \\
\hline Surrogate Recovery (\%) & & & & & & \\
\hline Bromofluorobenzene & $\%$ & 98 & N/A & 79 & N/A & 1057747 \\
\hline $\begin{array}{l}\text { D4-1,2-Dichlorcethane } \\
\text { D8-Toluene }\end{array}$ & $\%$ & 94 & N/A & 97 & N/A & 1057747 \\
\hline $\begin{array}{l}\text { ND = Not detected } \\
\text { N/A = Not Applicable } \\
\text { RDL = Reportable Detection Limit } \\
\text { QC Batch = Quality Control Batch }\end{array}$ & $\%$ & 97 & N/A & 91 & N/A & 1057747 \\
\hline
\end{tabular}




\begin{tabular}{|c|c|c|c|c|c|c|c|}
\hline & Units & $\begin{array}{l}\text { VOC-1 } \\
\text { QB-B-2 } \\
9 / 17 / 106 \\
\end{array}$ & RDL & QC Batch & $\begin{array}{c}\text { VOC-2 } \\
\text { QB-B-1\&2 } \\
9 / 17 / 106 \\
\end{array}$ & $\mathrm{RDL}$ & QC Batch \\
\hline Vinyl Chloride & ug & ND & 0.01 & 1057747 & ND & 0.1 & 1058299 \\
\hline 1,1-Dichloroethane & ug & $\mathrm{ND}$ & 0.01 & 1057747 & $\mathrm{ND}$ & 0.1 & 1058299 \\
\hline Chloroform & ug & 0.03 & 0.01 & 1057747 & ND & 0.1 & 1058299 \\
\hline 1,2-Dichloroethane & ug & ND & 0.007 & 1057747 & $\mathrm{ND}$ & 0.07 & 1058299 \\
\hline Methyl Ethyl Ketone (2-Butanone) & ug & ND & 0.04 & 1057747 & 0.5 & 0.4 & 1058299 \\
\hline Carbon Tetrachloride & ug & ND & 0.02 & 1057747 & ND & 0.2 & 1058299 \\
\hline Benzene & ug & 0.371 & 0.009 & 1057747 & 1.48 & 0.09 & 1058299 \\
\hline Trichloroethylene & ug & $\mathrm{ND}$ & 0.01 & 1057747 & ND & 0.1 & 1058299 \\
\hline Tetrachloroothylene & ug & ND & 0.02 & 1057747 & $\mathrm{ND}$ & 0.2 & 1058299 \\
\hline Chlorobenzene & ug & ND & 0.01 & 1057747 & $\mathrm{ND}$ & 0.1 & 1058299 \\
\hline \multicolumn{8}{|l|}{ Surrogate Recovery (\%) } \\
\hline Bromofluorobenzene & $\%$ & 107 & $\mathrm{~N} / \mathrm{A}$ & 1057747 & 95 & N/A & 1058299 \\
\hline D4-1,2-Dichloroethane & $\%$ & 95 & $\mathrm{~N} / \mathrm{A}$ & 1057747 & 111 & N/A & 1058299 \\
\hline D8-Toluene & $\%$ & 92 & N/A & 1057747 & 105 & N/A & 1058299 \\
\hline \multicolumn{8}{|l|}{$\begin{array}{l}\text { ND = Not detected } \\
\text { N/A = Not Applicable } \\
\text { RDL = Reportable Detection Limit } \\
\text { QC Batch = Quality Control Batch }\end{array}$} \\
\hline
\end{tabular}

\subsection{TCLP Pollutants in Fly Ash and Bottom Ash Generated During Tests in the}

\section{Laboratory-Scale Incinerator}

The fly ash and bottom ash from samples NQ-AD and QB-B were subjected to TCLP analysis for trace metals, VOCs and semi-VOCs. It is found that trace metals, VOCs and semi-VOCs in the fly ash are always undetectable or very close to the instrument (ICP-MS, GC-MS) detection limits. Tables 5 and 6 list the results for trace metals, Table 7 lists the results for VOC leachate in fly ash, and Table 8 lists the results for semi-VOCs in fly ash by TCLP. 
Table 5. Trace Metals in Fly Ash from Waste Pellets after Incineration by TCLP

\begin{tabular}{|c|c|c|c|c|c|c|c|c|c|c|c|}
\hline Trace metals in Fly ash & $\mathrm{Si}$ & Sr & $\mathrm{Ag}$ & $\mathrm{Fe}$ & As & $\mathrm{Cd}$ & $\mathrm{Cr}$ & $\mathrm{Cu}$ & $\mathrm{Ni}$ & $\mathrm{Pb}$ & $\mathrm{Hg}$ \\
\hline unit & \multicolumn{10}{|c|}{$\mathrm{ppm}$} & $\mathrm{ppb}$ \\
\hline $\begin{array}{l}\text { NQ-AD (100 grams fly ash } \\
\text { subjected to TCLP } \\
\text { procedure with } 2000 \mathrm{ml} \\
\text { extraction solution) }\end{array}$ & 13.8 & 1309 & 0.028 & 0.006 & UD & UD & UD & 0.161 & 0.542 & UD & 1.5 \\
\hline $\begin{array}{l}\text { QB-B Fly Ash (3 grams fly } \\
\text { ash subjected to TCLP } \\
\text { procedure with } 80 \mathrm{ml} \\
\text { extraction solution) }\end{array}$ & 33.2 & 18 & 0.007 & UD & UD & UD & UD & 0.033 & 0.175 & UD & 1.7 \\
\hline Detection limit, in ppb & 1.4 & 0.01 & 5 & 3 & 9.3 & 3 & 0.15 & 0.3 & 0.3 & 5.8 & 0.1 \\
\hline
\end{tabular}

Note: 1. Not enough fly ash of Sample QB-B can be generated as equired because of fly ash emission is very low

2. Estimated generation rate of fly ash from Sample NQ-AD $=0.065 \mathrm{~g} / \mathrm{g}$ raw waste pellet, thus, $100 \mathrm{~g}$ fly ash $/ 1.5 \mathrm{Kg}$ raw waste pellets

3. Estimated generation rate of fly ash from Sample $Q B-B=0.0026 \mathrm{~g} / \mathrm{g}$ raw waste pellet, thus, $100 \mathrm{~g}$ fly ash $/ 40 \mathrm{Kg}$ raw waste pellets 4. Sampling:EPA method 29, TCLP: EPA method 1311, Analysis: EPA 200.7

Table 6. Trace Metals in Bottom Ash from Waste Pellets after Incineration by TCLP

\begin{tabular}{|c|c|c|c|c|c|c|c|c|c|c|c|}
\hline Trace metals in Bottom ash & $\mathrm{Si}$ & $\mathrm{Sr}$ & $\mathrm{Ag}$ & $\mathrm{Fe}$ & As & $\mathrm{Cd}$ & $\mathrm{Cr}$ & $\mathrm{Cu}$ & $\mathrm{Ni}$ & $\mathrm{Pb}$ & $\mathrm{Hg}$ \\
\hline $\begin{array}{c}\text { unit } \\
\end{array}$ & \multicolumn{10}{|c|}{$\mathrm{ppm}$} & $\mathrm{ppb}$ \\
\hline $\begin{array}{l}\text { NQ-AD Bottom ash }(100 \\
\text { grams bottom ash subjected } \\
\text { to TCLP procedure with } \\
2000 \mathrm{ml} \text { extraction solution) }\end{array}$ & 219 & 574 & 0.012 & 0.009 & UD & UD & UD & 0.041 & 0.191 & UD & 0.67 \\
\hline $\begin{array}{l}\text { QB-B Bottom Ash ( } 100 \\
\text { grams bottom ash subjected } \\
\text { to TCLP procedure with } \\
2000 \mathrm{ml} \text { extraction solution) }\end{array}$ & 8.67 & 7.8 & UD & 0.007 & UD & UD & UD & 0.181 & 0.174 & UD & 0.46 \\
\hline \multirow[t]{2}{*}{ Detection limit } & & & & & & $\mathrm{ppb}$ & & & & & \\
\hline & 1.4 & 0.01 & 5 & 3 & 9.3 & 3 & 0.15 & 0.3 & 0.3 & 5.8 & 0.1 \\
\hline
\end{tabular}

Note: 1. Estimated generation rate of bottom ash from Sample NQ-AD $=0.935 \mathrm{~g} / \mathrm{g}$ raw waste pellet, thus, $100 \mathrm{~g}$ bottom ash $/ 0.107 \mathrm{Kg}$ raw waste pellets 2. Estimated generation rate of bottom ash from Sample QB-B $=0.997 \mathrm{~g} / \mathrm{g}$ raw waste pellet, thus, $100 \mathrm{~g}$ bottom ash $/ 0.1003 \mathrm{Kg}$ raw waste pellets 3. Sampling:EPA method 29, TCLP: EPA method 1311, EPA 200.7 
Table 7. VOC in Fly Ash from Waste Pellets after Incineration by TCLP

\begin{tabular}{|c|c|c|c|c|}
\hline \multicolumn{5}{|l|}{ QB-B fly ash } \\
\hline & Method & Results & Unit & Detection limit \\
\hline 1,1-Dichloroethene & EPA $8260 \mathrm{~B}$ & 0.05, UD & $\mathrm{mg} / \mathrm{l}$ & 0.05 \\
\hline 1,2-Dichloroethane & EPA $8260 \mathrm{~B}$ & 0.05, UD & $\mathrm{mg} / \mathrm{l}$ & 0.05 \\
\hline 2-Butanone & EPA $8260 \mathrm{~B}$ & 0.05, UD & $\mathrm{mg} / \mathrm{l}$ & 0.05 \\
\hline Benzene & EPA $8260 \mathrm{~B}$ & 0.05 , UD & $\mathrm{mg} / \mathrm{l}$ & 0.05 \\
\hline Carbon Tetrachloride & EPA $8260 \mathrm{~B}$ & 0.05, UD & $\mathrm{mg} / \mathrm{l}$ & 0.05 \\
\hline Chlorobenzene & EPA $8260 \mathrm{~B}$ & 0.05, UD & $\mathrm{mg} / \mathrm{l}$ & 0.05 \\
\hline Chloroform & EPA $8260 \mathrm{~B}$ & 0.05, UD & $\mathrm{mg} / \mathrm{l}$ & 0.05 \\
\hline Tetrachloroethene & EPA $8260 \mathrm{~B}$ & 0.05, UD & $\mathrm{mg} / \mathrm{l}$ & 0.05 \\
\hline Trichloroethene & EPA $8260 \mathrm{~B}$ & 0.05, UD & $\mathrm{mg} / \mathrm{l}$ & 0.05 \\
\hline Vinyl Chloride & EPA $8260 \mathrm{~B}$ & $0.05, \mathrm{UD}$ & $\mathrm{mg} / \mathrm{l}$ & 0.05 \\
\hline \multicolumn{5}{|l|}{ NQ-AD fly ash } \\
\hline & Method & Results & Unit & Detection limit \\
\hline 1,1-Dichloroethene & EPA $8260 \mathrm{~B}$ & 0.05, UD & $\mathrm{mg} / \mathrm{l}$ & 0.05 \\
\hline 1,2-Dichloroethane & EPA $8260 \mathrm{~B}$ & 0.05 , UD & $\mathrm{mg} / \mathrm{l}$ & 0.05 \\
\hline 2-Butanone & EPA $8260 \mathrm{~B}$ & 0.05, UD & $\mathrm{mg} / \mathrm{l}$ & 0.05 \\
\hline Benzene & EPA $8260 \mathrm{~B}$ & 0.05 , UD & $\mathrm{mg} / \mathrm{l}$ & 0.05 \\
\hline Carbon Tetrachloride & EPA $8260 \mathrm{~B}$ & 0.05, UD & $\mathrm{mg} / \mathrm{l}$ & 0.05 \\
\hline Chlorobenzene & EPA $8260 \mathrm{~B}$ & 0.05, UD & $\mathrm{mg} / \mathrm{l}$ & 0.05 \\
\hline Chloroform & EPA $8260 \mathrm{~B}$ & 0.05, UD & $\mathrm{mg} / \mathrm{l}$ & 0.05 \\
\hline Tetrachloroethene & EPA $8260 \mathrm{~B}$ & 0.05 , UD & $\mathrm{mg} / \mathrm{l}$ & 0.05 \\
\hline Trichloroethene & EPA $8260 \mathrm{~B}$ & 0.05, UD & $\mathrm{mg} / \mathrm{l}$ & 0.05 \\
\hline Vinyl Chloride & EPA $8260 \mathrm{~B}$ & 0.05, UD & $\mathrm{mg} / \mathrm{l}$ & 0.05 \\
\hline
\end{tabular}

Table 8. Semi-VOC in Fly Ash from Waste Pellets after Incineration by TCLP

\begin{tabular}{ccccc}
\hline QB-B fly ash & Method & Results & Unit & Detection limit \\
\hline 1,4-Dichlorobenzene & EPA 8270 D & UD & $\mathrm{mg} / \mathrm{l}$ & 0.05 \\
2,4-Dinitrotoluene & EPA 8270 D & UD & $\mathrm{mg} / \mathrm{l}$ & 0.05 \\
Hexachlorobenzene & EPA 8270 D & UD & $\mathrm{mg} / \mathrm{l}$ & 0.05 \\
Hexachlorobutadiene & EPA 8270 D & UD & $\mathrm{mg} / \mathrm{l}$ & 0.05 \\
Hexachloroethane & EPA 8270 D & UD & $\mathrm{mg} / \mathrm{l}$ & 0.05 \\
Nitrobenzene & EPA 8270 D & UD & $\mathrm{mg} / \mathrm{l}$ & 0.05 \\
Pentachlorophenol & EPA 8270 D & UD & $\mathrm{mg} / \mathrm{l}$ & 0.05 \\
Pyridine & EPA 8270 D & UD & $\mathrm{mg} / \mathrm{l}$ & 0.05 \\
2,4,5-Trichlorophenol & EPA 8270 D & UD & $\mathrm{mg} / \mathrm{l}$ & 0.05 \\
2,4,6-Trichlorophenol & EPA 8270 D & UD & $\mathrm{mg} / \mathrm{l}$ & 0.05 \\
& & & & \\
\hline NQ-AD fly ash & & & & \\
& Method & Results & $\mathrm{Unit}$ & Detection limit \\
\hline 1,4-Dichlorobenzene & EPA 8270 D & UD & $\mathrm{mg} / \mathrm{l}$ & 0.05 \\
2,4-Dinitrotoluene & EPA 8270 D & UD & $\mathrm{mg} / \mathrm{l}$ & 0.05 \\
Hexachlorobenzene & EPA 8270 D & UD & $\mathrm{mg} / \mathrm{l}$ & 0.05 \\
Hexachlorobutadiene & EPA 8270 D & UD & $\mathrm{mg} / \mathrm{l}$ & 0.05 \\
Hexachloroethane & EPA 8270 D & UD & $\mathrm{mg} / \mathrm{l}$ & 0.05 \\
Nitrobenzene & EPA 8270 D & UD & $\mathrm{mg} / \mathrm{l}$ & 0.05 \\
Pentachlorophenol & EPA 8270 D & UD & $\mathrm{mg} / \mathrm{l}$ & 0.05 \\
Pyridine & EPA 8270 D & UD & $\mathrm{mg} / \mathrm{l}$ & 0.05 \\
2,4,5-Trichlorophenol & EPA 8270 D & UD & $\mathrm{mg} / \mathrm{l}$ & 0.05 \\
2,4,6-Trichlorophenol & EPA 8270 D & UD & $\mathrm{mg} / \mathrm{l}$ & 0.05 \\
\hline
\end{tabular}




\section{CONCLUSIONS}

During this quarter, the following progress has been made:

a) The installation of CFBC Facility was continued. All ash recirculation ducts have been aligned and installed. Both loop seals have been installed in ash recirculation path. Cold ash supply duct has been installed. Installation of the induced draft fan power supply and variable speed drive has been completed. Wind box installation has been completed. Deliveries of some parts were delayed and proposed tests were be postponed accordingly.

b) A laboratory-scale waste incineration test was conducted with two waste pellets and various emissions were measured.

\section{FUTURE WORK AND UPDATED SCHEDULE}

\subsection{Future Work}

During the next quarter, work will focus on the following activities:

a) Install one remaining thermal expansion joint below the lower loop seal.

b) Design, order components and install pneumatic control elements and signal lines for loop seal operation.

c) Install cold ash bunker and transport auger, drive, power supply and control.

d) Install induced draft fan, including support structure.

e) Install flue gas ductwork between secondary cyclone and ID fan inlet and flue duct on discharge side of ID fan.

f) Install secondary combustion air fan, fabricate distribution duct work and install secondary air flow control valves and their control elements.

g) Specify, order and install pressure and flow transducers, modifying some ports where building support structure interferes.

h) Design, specify, purchase and install sensor and control signal interface hub enclosures within combustor tower facility. Install digital data lines from these hubs to the process control computer.

i) Install control elements for pneumatic operation of primary combustion/fluidizing air diverter valve operation. 
j) Complete flexible duct, power supply, control elements and enclosure installation for bed pre-heater operation.

k) Perform so called "leak test" or cold fluidization test to confirm proper operation of sub-systems from the combustion/fluidization air supply to the induced fan. For this test, pressure and flow sensors will be in operation. CFBC ash obtained from another commercial facility will be used in this test.

\subsection{Project Schedule}

Based on the current status of the project, the project schedule for the remainder work is shown in Table 9.

Table 9. Project schedule

\begin{tabular}{|c|c|}
\hline Task & Schedule \\
\hline $\begin{array}{l}\text { Install induced draft fan and flue ducts to and from; } \\
\text { construct machine base mount }\end{array}$ & February 15, 2007 \\
\hline $\begin{array}{l}\text { Install one remaining thermal expansion joint on ash } \\
\text { duct below lower loop seal }\end{array}$ & February 15, 2007 \\
\hline Install both loop seal operators and control lines & February 15, 2007 \\
\hline $\begin{array}{l}\text { Install ash transport auger, seals and bearings, gear } \\
\text { motor, variable speed drive, power supply, control } \\
\text { interface }\end{array}$ & February 15, 2007 \\
\hline Perform leak test of System & February 15, 2007 \\
\hline $\begin{array}{l}\text { Install three gravimetric supply bunkers and associated } \\
\text { supply duct components. }\end{array}$ & April 30, 2007 \\
\hline $\begin{array}{l}\text { Install secondary combustion air fan and distribution } \\
\text { duct work; control interface }\end{array}$ & April 30, 2007 \\
\hline $\begin{array}{l}\text { Complete ordering and installation of coolant system } \\
\text { components }\end{array}$ & June 30, 2007 \\
\hline
\end{tabular}




\begin{tabular}{|l|l|}
\hline $\begin{array}{l}\text { Complete specification, ordering of components and } \\
\text { installation of water treatment system for heat exchanger } \\
\text { coolant loop }\end{array}$ & June 30,2007 \\
\hline $\begin{array}{l}\text { Complete specification and installation of sensors and } \\
\text { control operators }\end{array}$ & July 31, 2007 \\
\hline $\begin{array}{l}\text { Complete process control and data acquisition computer } \\
\text { program development }\end{array}$ & August 31 2007 \\
\hline $\begin{array}{l}\text { Complete the installation of the CFBC } \\
\text { Facility }\end{array}$ & August 31,2007 \\
\hline $\begin{array}{l}\text { Based on the experimental data obtained from the } \\
\text { laboratory-scale CFBC Facility, determine the optimal } \\
\text { conditions for co-firing waste materials with high sulfur } \\
\text { coals in the CFBC Facility. }\end{array}$ & September 30,2007 \\
\hline $\begin{array}{l}\text { Complete the study to determine the effect of air staging, } \\
\text { fuel feeding position, and limestone feeding on the } \\
\text { gaseous emissions in the CFBC Facility. }\end{array}$ & September 30,2007 \\
\hline $\begin{array}{l}\text { Complete the investigation of mercury emissions from } \\
\text { co-firing of waste materials with high sulfur coal in the } \\
\text { CFBC Facility }\end{array}$ & September 30, 2007 \\
\hline \begin{tabular}{l} 
Submit final report \\
\hline
\end{tabular} & October 31, 2007 \\
\hline
\end{tabular}

\section{ACRONYMS AND ABBREVIATIONS}

$\begin{array}{ll}\text { CFBC } & \text { Circulating Fluidized-Bed Combustion } \\ \text { DOE } & \text { U.S. Department of Energy } \\ \text { ECTL } & \text { Environmental Control Technology Laboratory } \\ \text { ISCET } & \text { Institute for Combustion Science and Environmental Technology } \\ \text { VOC } & \text { Volatile Organic Compounds } \\ \text { semi-VOC } & \text { Semi-volatile Organic Compounds } \\ \text { TCLP } & \text { Toxicity Characteristic Leaching Procedure }\end{array}$

